reviews and mini-reviews on particularly timely or high-impact issues, as well as encouraging thoughtful commentary on the issues shaping the field of microbial ecology. And most importantly, we seek to publish high-quality studies in microbial ecology and contributions of broad biological interest and impact.

It is being increasingly realized that the world is to a large extent the domain of microbial ecology and we hope to make The ISME Journal the vehicle to express the emerging and exciting research that continues to bring this revelation to the fore!
GA Kowalchuk is at Netherlands Institute of Ecology, Centre for Terrestrial Ecology Netherlands,

Heteren, The Netherlands;

JF Heidelberg is at University of Southern California,

Wrigley Marine Science Center, CA, USA and

MJ Bailey is at Centre for Ecology \&

Hydrology - Oxford, Institute of Virology and

Environmental Microbiology,

Oxford, UK.

E-mail: g.kowalchuk@nioo.knaw.nl

\title{
The ISME Journal: a major milestone for the International Society for Microbial Ecology and the microbial ecology community
}

\author{
Yehuda Cohen, Staffan Kjelleberg and Hilary M Lappin-Scott
}

The ISME Journal (2007) 1, 3-4; doi:10.1038/ismej.2007.14

The launch of The ISME Journal - a Multidisciplinary Journal of Microbial Ecology marks the maturation of the multidisciplinary field of microbial ecology. Our vision is to contribute significantly to our understanding of the behavior of microorganisms in their environment: unraveling the underlining mechanisms of interactions among prokaryotes, with their eukaryotic partners and abiotic environments, and deciphering the means by which microorganisms turn the wheels of planet Earth.

Our aim is that The ISME Journal will operate at the leading edge of publications and include original contributions, as well as reviews and commentaries expressing the scientific excitement generated from studies of both basic concepts in microbial ecology and their many applications. It is envisaged that an enhanced understanding of the fundamentals in microbial ecology is also essential for the well-being and the future of the planet we inhabit.

This is the first journal fully owned by the International Society for Microbial Ecology and it was established to provide the professional platform for an active and rapidly expanding scientific community in the multidisciplinary field of microbial ecology. We foresee that The ISME Journal will become a vehicle tuned to the ever- evolving needs of our scientific community through close interactions between the editors, the editorial board and the advisory committee of the journal, the Society and our scientific community. These interactions will also be facilitated by the biannual international symposia, international and regional workshops and our interactive web site www. microbes.org. We therefore encourage the readers, whether established researchers, young scientists or research students, to engage with us and provide suggestions and comments on how to arrive at our mutual goal of establishing a truly excellent and leading journal in microbial ecology. In seeking excellence, we selected the Nature Publishing Group (NPG) to act as the publisher of The ISME Journal, and we value the open, welcoming and fruitful interactions we have experienced with the highly professional team of NPG. ISME is pleased to be part of the Nature family and we trust that, with the cooperation of the broader scientific community of basic and applied scientists, The ISME Journal will become the key vehicle, and publication of choice, for disseminating new and important findings in our multidisciplinary research arena.

The ISME Society is less than 10 years old and was established in 1998 by the combined visionary efforts of Jim Tiedje and Usio Simidu, who served as consecutive Chairs of the International Committee for Microbial Ecology (ICOME), which was under the auspices of the International Union of 
Microbiology Societies (IUMS). As an independent society, ISME has organized highly successful international symposia in Halifax (1998), Amsterdam (2001), Cancun (2004) and Vienna (2006). The number of participants in our symposia continues to increase, reflecting the surge in interest in microbial ecology, from 300 participants in Dunedin, New Zealand in 1977 for the inaugural meeting of ICOME, to an impressive gathering of more than 2000 attendees at ISME-11 in Vienna last year. This meeting attracted participants from 65 countries representing all geographic regions. We especially cherish the ongoing participation of a large number of graduate students and young scientists at our symposia, indicating a very healthy future of ISME and the discipline of microbial ecology.

The field of microbial ecology does not adhere to boarders and requires effective cooperation to foster discipline at the international level. It is the objective of The ISME Journal to facilitate these interactions. We sincerely hope that the ISME Journal will serve as an important platform to promote microbial ecology to the scientific community at large and also effectively clarify and inform the broader community and decision makers of the pivotal role of microorganisms in the well-being of life on Earth and our communal future.

$Y$ Cohen (ISME President 2001-2004), is at the Faculty of Science, The Alexander Silberman Institute of Life Sciences, Hebrew University of Jerusalem, Jerusalem, Israel. E-mail: yehucoh@vms.huji.ac.il; $S$ Kjelleberg (ISME President 2004-2006), is at the School of Biotechnology and Biomolecular Sciences, University of New South Wales, Sydney, New South Wales, Australia. E-mail: skjelleberg@science.unsw.edu.au and HM Lappin-Scott (ISME President 2006-2008), is at the School of Biosciences, University of Exeter, Exeter, Devon, UK. E-mail: H.M.Lappin-Scott@exeter.ac.uk

\title{
Microbial ecology and environmental biotechnology
}

\author{
Willy Verstraete
}

The ISME Journal (2007) 1, 4-8; doi:10.1038/ismej.2007.7

\section{The domain}

Environmental biotechnology deals with the microbiota in the water, air and soil surrounding us; moreover, the gastrointestinal tract, that is the 'outside world inside the living animals' is also often considered to be part of the environmental biotechnology domain (Figure 1). In these open systems, the microbial communities are complex and constantly changing. One can consider these communities as accidental assemblages or as metagenomic functional units (Tringe et al., 2005). An important issue is that they represent superb resources in terms of genes and functionalities. Yet, there is much more possible and necessary than the mere listing of DGGE/T-RFLP/clone libraries. The key point for the next decades is to deal with the proper management of these microbial resources. At all levels of our society, the concept of human resource management has become of preponderant importance and plenty of top-ranking business schools develop it; in the domain of environmental biotechnology, the focus for the next decades must be on microbial resource management (MRM).

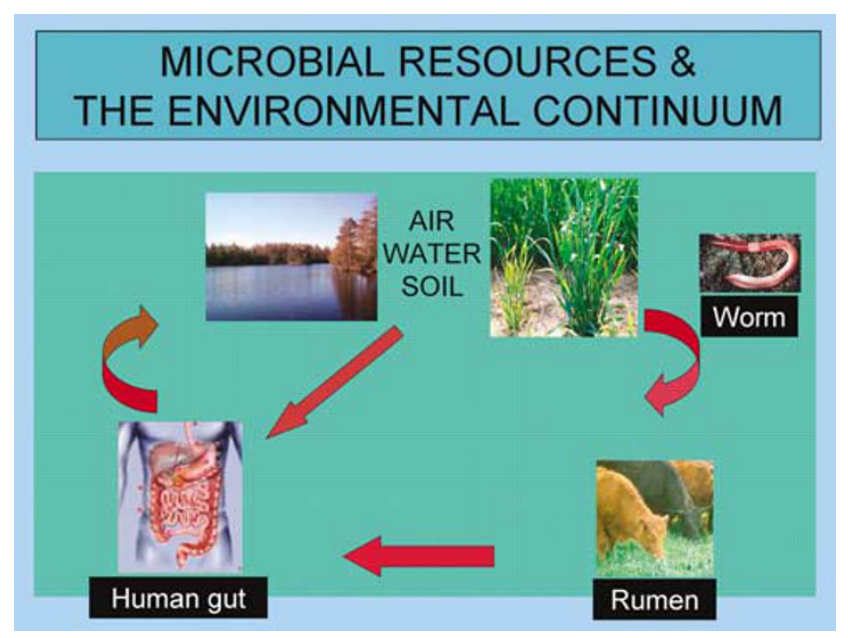

Figure 1 Schematic representation of the various domains covered by environmental biotechnology. 